\title{
Referred cutaneous allodynia in a migraine patient without simultaneous headache
}

\author{
Avi Ashkenazi \\ Thomas Jefferson University \\ Carla LoPinto \\ Thomas Jefferson University \\ William B. Young \\ Thomas Jefferson University
}

Follow this and additional works at: https://jdc.jefferson.edu/neurologyfp

Part of the Neurology Commons

Let us know how access to this document benefits you

\section{Recommended Citation}

Ashkenazi, Avi; LoPinto, Carla; and Young, William B., "Referred cutaneous allodynia in a migraine patient without simultaneous headache" (2005). Department of Neurology Faculty Papers. Paper 8.

https://jdc.jefferson.edu/neurologyfp/8

This Article is brought to you for free and open access by the Jefferson Digital Commons. The Jefferson Digital Commons is a service of Thomas Jefferson University's Center for Teaching and Learning (CTL). The Commons is a showcase for Jefferson books and journals, peer-reviewed scholarly publications, unique historical collections from the University archives, and teaching tools. The Jefferson Digital Commons allows researchers and interested readers anywhere in the world to learn about and keep up to date with Jefferson scholarship. This article has been accepted for inclusion in Department of Neurology Faculty Papers by an authorized administrator of the Jefferson Digital Commons. For more information, please contact: JeffersonDigitalCommons@jefferson.edu. 


\title{
Referred cutaneous allodynia in a migraine patient without simultaneous headache
}

\author{
Avi Ashkenazi, M.D., Carla LoPinto, B.A., and William B. Young, M.D. \\ Jefferson Headache Center
}

\section{Introduction}

Cutaneous allodynia is defined as the perception of pain when a non-noxious stimulus is applied to normal skin (1). This phenomenon has been demonstrated in migraine patients during an acute attack (2). It is thought to result from central sensitization of neurons in the trigemino-vascular system and may spread to areas beyond that of the referred head pain $(2,3)$.

In a recent study, migraine patients demonstrated increased temporal summation to painful mechanical stimuli in their referred head pain area between attacks (4). This suggests that changes in physiologic properties of nociceptive neurons may occur in migraine patients between attacks.

We describe a migraine patient with interictal cutaneous allodynia contralateral to her usual head pain.

\section{Case Report}

The patient is a 39-year-old female with a history of episodic migraine with aura since age 12, who came to the Jefferson Headache Center for a routine visit. She had an average of six migraine attacks per month. Her attacks were characterized by a throbbing pain in the right frontal area that was exacerbated by tilting her head, coughing and climbing stairs. She also noticed that touching her face, combing her hair and wearing anything on her head (e.g. a hat or jewelry) were bothersome during her headaches. The headache was accompanied by nausea, photophobia, phonophobia and osmophobia. The 
average pain severity was 8 (on an 11-point verbal scale). The attacks lasted for an average of 48 hours. Once every two months, a visual aura preceded her migraine attacks. The aura lasted approximately five minutes. Her last migraine attack occurred six days prior to her visit.

The patient was taking topiramate $200 \mathrm{mg} /$ day, verapamil $480 \mathrm{mg} /$ day and riboflavin 400 $\mathrm{mg}$ /day for migraine prophylaxis. She noted paresthesias of the fingertips as a side effect of topiramate treatment.

At the time of her visit, the patient did not report any head pain or associated symptoms and her physical examination was normal.

\section{Methods}

The patient was tested for both static mechanical (pressure) and dynamic mechanical (brush) cutaneous allodynia. Brush allodynia was tested by gently applying a $4 \times 4$ gauze pad to the skin ten times at a frequency of $2 / \mathrm{sec}$ as we described previously (5). Pressure allodynia was tested by applying Von Frey Hairs (VFH) (Touch-Test ${ }^{\text {TM }}$ Sensory Evaluators, North Coast Medical, Inc.) of two different sizes, 4.93 (8 g) and 5.88 (60 g) three times for each area, holding down for $1 \mathrm{sec}$ and releasing (since there are no standardized threshold pressure levels that cause pain in normal subjects, the selection of these weights was somewhat arbitrary). The areas tested for both brush and pressure allodynia were the forehead $\left(\mathrm{V}_{1}\right)$, posterior neck $\left(\mathrm{C}_{2}, \mathrm{C}_{3}\right)$ and inner forearm $\left(\mathrm{C}_{8}\right)$, bilaterally. The degree of allodynia was measured using a $100 \mathrm{~mm}$ visual analog scale (VAS).

\section{Results}

Brush allodynia was noted when the gauze was applied to the left posterior neck and the left forehead (55 $\mathrm{mm}$ and $57 \mathrm{~mm}$ on the VAS, respectively). Interestingly, the evoked pain was on the right forehead (the area of her usual migraine head pain), but not at the 
stimulated areas, in both instances (Figure 1). No allodynia was noted when the gauze was applied directly to the right forehead or to the right posterior neck.

Allodynia was also noted when a VFH of either size was applied to the left posterior neck ( $34 \mathrm{~mm}$ and $35 \mathrm{~mm}$ on the VAS for the 4.93 and 5.88 sized filaments, respectively). Again, the evoked pain was perceived in the right forehead but not at the stimulated area (Figure 2), and no allodynia was noted when pressure was applied directly to the right forehead or to the right posterior neck.

\section{Discussion}

This patient demonstrates several interesting aspects of cutaneous allodynia. First, she had allodynia between migraine attacks; second, she had allodynia at a different site than the stimulated area; third, her brush and pressure allodynia had different patterns of expression. The cutaneous allodynia in this patient may have been constant, as we found it to occur between attacks. However, since we did not repeat the allodynia testing at a later time we can not make this conclusion with certainty.

Cutaneous allodynia in migraine and in other pain syndromes is thought to result from central sensitization of neurons in the trigeminal nucleus caudalis (TNC) or in the spinal dorsal horn $(1,3,6)$. In migraine, this is supported by experimental data showing changes in the physiological properties of central trigeminal neurons after chemical irritation of the dura (7). The finding in this patient of allodynia between attacks suggests that neuronal sensitization in migraine may last longer than the acute attack. It has been suggested that central sensitization of spino-thalamic tract neurons after intra-dermal injection of capsaicin is a form of long-term potentiation (1). This may result from activation of signal transduction cascades, involving a number of protein kinases (8). We speculate that these changes may occur also in TNC neurons in migraine patients between attacks. In a recent study, migraine patients were found to have increased pain scores in temporal summation tests for both mechanical and electrical stimuli (4). They also had a slightly lowered pain threshold for mechanical stimulation. These findings 
show that migraine patients tend to exhibit wind-up more than non-migraineur individuals.

Although referred pain causing allodynia has been described (9), a non-noxious mechanical stimulus causing pain referred only to a separate location from that stimulated has not been previously documented in migraine. We suggest the term "referred allodynia" for this phenomenon.

The mechanism by which this patient perceived the allodynic pain contralaterally to the area of her usual migraine pain is undetermined. Pain arising at sites contralateral to the pathological area has been described in various conditions, including reflex sympathetic dystrophy (10), causalgia (11) and atypical facial pain (12). This has been termed mirrorimage neuropathic pain and is associated with mechanical allodynia. A similar phenomenon has been described in the sciatic inflammatory neuropathy animal model (13). In this model, intense immune activation of the sciatic nerve produces bilateral (ipsilateral and mirror-image) allodynia. The allodynia in this model was shown to be mediated by spinal glial and pro-inflammatory cytokines.

In migraine, the spatial distribution of pain and allodynia may not exactly overlap. Burstein et al have shown that migraine patients in the acute attack may have cutaneous allodynia in a larger area than that of the actual head pain (2). The fact that this patient perceived pain contralaterally to the stimulated area, in the area of her usual head pain (right frontal), suggests sensitization of TNC neurons which are active during her usual migraine attacks. This implies a connection between sensory pathways of the two sides via commissural fibers, either at the TNC or at higher levels. Since most fibers carrying sensory information from the face cross the midline at the TNC level, this may also be the site of sensitization. Neurophysiologic studies are needed, however, to confirm this hypothesis.

Although cutaneous allodynia is mostly associated with central sensitization, it is possible that peripheral sensitization of cutaneous receptors at the cervical and frontal areas occurred in this patient and caused the increased skin sensitivity to mechanical stimuli. The fact that areas contralateral to the stimulus showed cutaneous allodynia points to a central mechanism for the allodynia, with a possible peripheral sensitization as well. 
The patient was under treatment with three migraine preventive drugs at the time of examination, topiramate, verapamil and riboflavin, which raises the concern that these drugs affected the results of her allodynia testing. We have not found, however, evidence in the literature for any of these drugs to causes or to relieve allodynia. In one study, verapamil, administered to rats with experimental tactile allodynia, had no effect on pain behavior (14).

This patient did not exhibit allodynia when stimulated on the side of her usual migraine pain. There is currently no satisfactory explanation to this observation. It may be attributed to a suppressor mechanism that is activated when sensory stimuli are applied to the skin ipsilateral to her head pain. The mechanism is, however, still unknown.

The fact that brush allodynia was experienced when stimulating the posterior neck and forehead while pressure allodynia was experienced when stimulating the posterior neck but not the forehead suggest that static and dynamic mechanical allodynia are independent phenomena and do not necessarily accompany each other in all skin areas. This observation is supported by previous studies, showing that allodynia to different sensory modalities may have different temporal and spatial profiles $(2,3)$.

In summary, this patient demonstrates cutaneous allodynia between migraine attacks, contralateral to the stimulated side. This suggests that sensitization of central neurons, with or without sensitization of peripheral neurons or receptors, in migraine may be long lasting. It also suggests that neurons or receptors contralateral to the stimulated area may be sensitized without simultaneous sensitization on the ipsilateral side. 


\section{References}

1. Willis WD. Role of neurotransmitters in sensitization of pain responses. Ann N Y Acad Sci 2001;933:142-56.

2. Burstein R, Yarnitsky D, Goor-Aryeh I, Ransil BJ, Bajwa ZH. An association between migraine and cutaneous allodynia.

Ann Neurol 2000;47(5):614-24.

3. Burstein R, Cutrer MF, Yarnitsky D. The development of cutaneous allodynia during a migraine attack clinical evidence for the sequential recruitment of spinal and supraspinal nociceptive neurons in migraine.

Brain 2000;123 ( Pt 8):1703-9.

4. Irit Weissman-Fogel, Elliot Sprecher, Yelena Granovsky and David Yarnitsky. Repeated noxious stimulation of the skin enhances cutaneous pain perception of migraine patients in-between attacks: clinical evidence for continuous subthreshold increase in membrane excitability of central trigeminovascular neurons. Pain 2003;104(3):693-700.

5. Ashkenazi A, Young WB. The effects of occipital nerve block on brush allodynia and pain in migraine. Headache 2003;43(5):543 [abstract]

6. Strassman AM, Raymond SA, Burstein R. Sensitization of meningeal sensory neurons and the origin of headaches. Nature 1996 12;384(6609):560-4.

7. Burstein R, Yamamura H, Malick A, Strassman AM. Chemical stimulation of the intracranial dura induces enhanced responses to facial stimulation in brain stem trigeminal neurons. J Neurophysiol 1998;79(2):964-82.

8. Lin Q, Peng YB, Willis WD. Possible role of protein kinase C in the sensitization of primate spinothalamic tract neurons. J Neurosci 1996 May 1;16(9):3026-34.

9. Nagaro T, Amakawa K, Arai T, Ochi G. Ipsilateral referral of pain following cordotomy. Pain 1993 ;55(2):275-6.

10. Maleki J, LeBel AA, Bennett GJ, Schwartzman RJ. Patterns of spread in complex regional pain syndrome, type I (reflex sympathetic dystrophy). Pain 2000 $1 ; 88(3): 259-66$. 
11. Shir Y, Seltzer Z. Effects of sympathectomy in a model of causalgiform pain produced by partial sciatic nerve injury in rats. Pain 1991;45(3):309-20.

12. Woda A, Pionchon P. A unified concept of idiopathic orofacial pain: pathophysiologic features. J Orofac Pain 2000;14(3):196-212.

13. Milligan ED, Twining C, Chacur M, Biedenkapp J, O'Connor K, Poole S, Tracey K, Martin D, Maier SF, Watkins LR. Spinal glia and proinflammatory cytokines mediate mirror-image neuropathic pain in rats. J Neurosci 2003 1;23(3):1026-40.

14. Chaplan SR, Pogrel JW, Yaksh TL. Role of voltage-dependent calcium channel subtypes in experimental tactile allodynia. J Pharmacol Exp Ther 1994;269(3):1117-23. 


\section{Legends}

Figure 1: Areas of brush stimulation (arrows) and area of referred allodynia (star).

Figure 2: Area of pressure stimulation (arrow) and area of referred allodynia (star).

Authors' final version prior to publication in Cephalalgia 25(1):75-78, January 2005.

Copyright $(\mathcal{C}$ by Blackwell Publishing, Inc. 

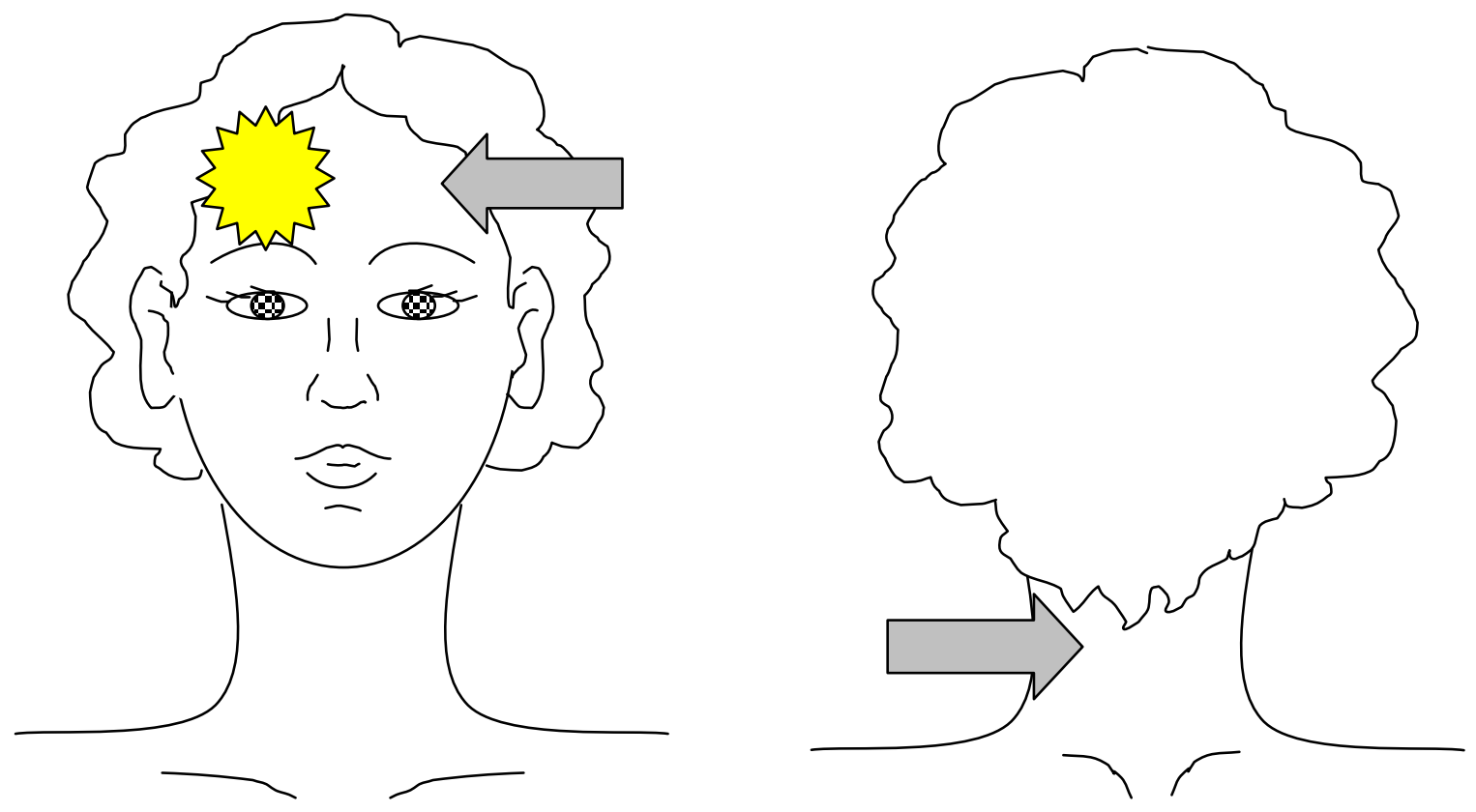

Figure 1
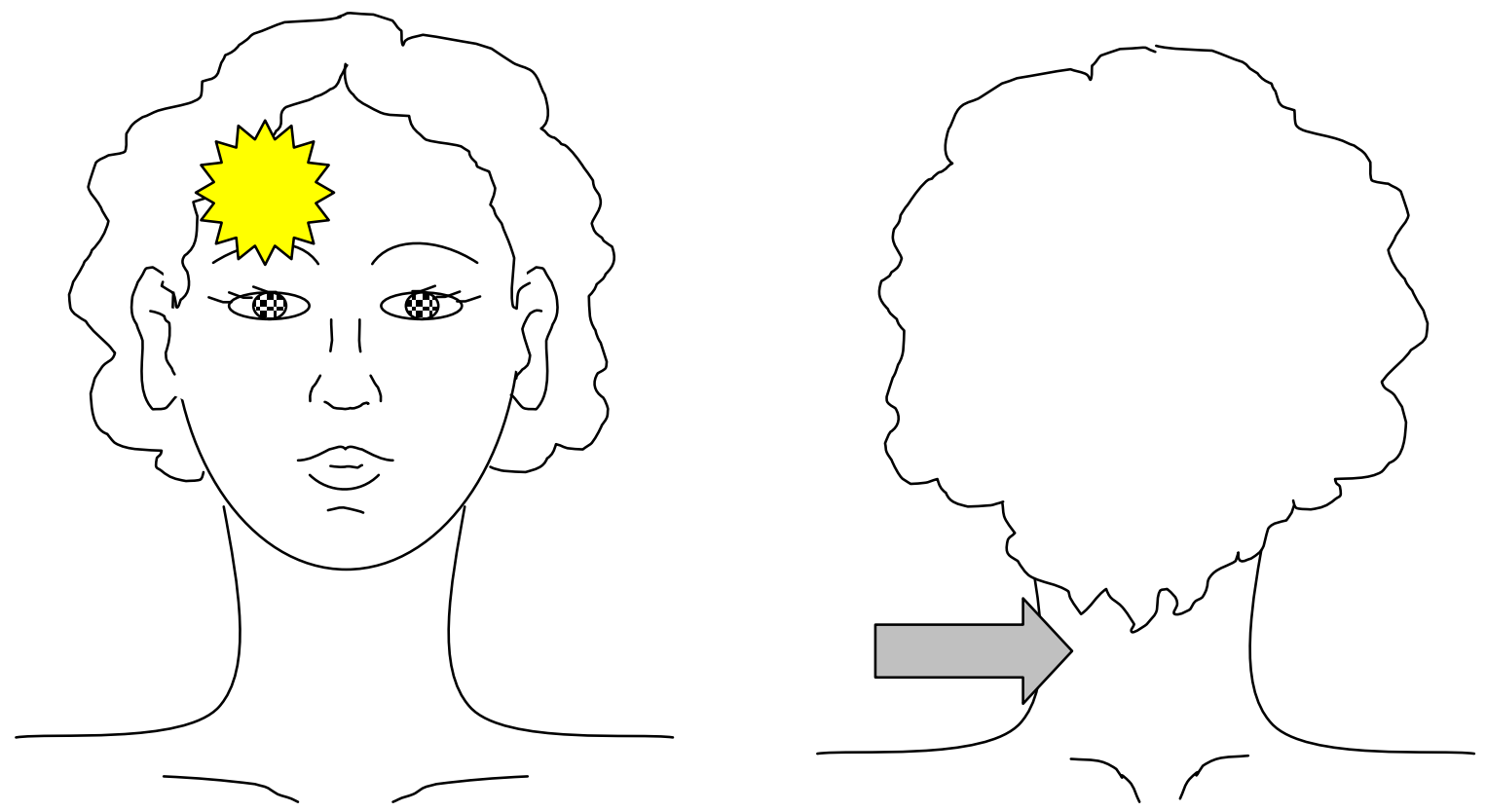

Figure 2

Authors' final version prior to publication in Cephalalgia 25(1):75-78, January 2005.

Copyright $(C)$ by Blackwell Publishing, Inc. 